\title{
Optical orientation and shifting of a single multiwalled carbon nanotube
}

\author{
Hongbao Xin and Baojun Li
}

The orientation and shifting of individual carbon nanotubes are extremely important in the assembly of building blocks of nanodevices and in the development of one-dimensional materials for interdisciplinary applications. Here, we report an optical method that is capable of producing the controlled orientation and targeted shifting of single multiwalled carbon nanotubes (MWCNTs) using an optical-fiber nanotip. In a demonstration of this technique, a single MWCNT with an outer diameter of $50 \mathrm{~nm}$ and a length of $0.9 \mu \mathrm{m}$ was first trapped by the nanotip using a laser beam with a wavelength of $980 \mathrm{~nm}$ and was then oriented and shifted along the nanotip axis as a result of the interaction of the MWCNT with the optical field output by the nanotip. Various optical powers were applied to characterize the orientation and shifting performance. The orientation and shifting of MWCNTs of various sizes were also demonstrated. Light: Science \& Applications (2014) 3, e205; doi:10.1038/lsa.2014.86; published online 26 September 2014

Keywords: carbon nanotube; nanomanipulation; optical orientation; optical shifting

\section{INTRODUCTION}

Carbon nanotubes (CNTs), including single-walled carbon nanotubes (SWCNTs) and multiwalled carbon nanotubes (MWCNTs), are considered to be promising building blocks for various applications because of their exceptional optical and electronic properties. $^{1,2}$ In particular, the integration of individual SWCNTs into highly ordered arrays can exert considerable influence on the performance of optoelectronic devices, ${ }^{3-5}$ and the integration of MWCNTs is also very important for a wide variety of applications, such as field emitters ${ }^{6}$ and chemical sensing. ${ }^{7}$ However, one of the most significant challenges for the integration of individual CNTs is the arrangement of CNTs with controlled orientation and placement. ${ }^{8-10}$ Various methods have been applied for the arrangement and manipulation of both individual SWCNTs and MWCNTs on substrates. For example, atomic force microscopy probes ${ }^{11}$ and the transfer-printing technique ${ }^{12}$ have been used for the arrangement and manipulation of SWCNTs, and scanning electron microscopes (SEMs) equipped with nano-manipulators ${ }^{13}$ and with inorganic nanoparticles as indicators ${ }^{14}$ have been used for the arrangement and manipulation of MWCNTs. These methods are applied for the manipulation of CNTs on a substrate, where the CNTs are not in random motion. In the case of CNTs suspended in liquid suspensions, their arrangement and manipulation would seem to be much more difficult because the CNTs are in random motion driven by the strong influence of Brownian motion. To manipulate and arrange CNTs in liquid suspensions, different methods have been applied. For example, dielectrophoresis ${ }^{15,16}$ has been used to manipulate and arrange SWCNTs, optoelectronic tweezers ${ }^{17}$ have been used to manipulate MWCNTs, and optical tweezers ${ }^{18}$ have been used to manipulate both SWCNTs and MWCNTs. For dielectrophoresis, microelectrodes that require elaborate fabrication using electron-beam lithography are needed. For the use of optoelectronic tweezers, both light and an electric bias are needed with the assistance of dielectrophoresis, and the single CNTs cannot be oriented and tend to shift with respect to the target locations. When optical tweezers are used, bundles of both SWCNTs and MWCNTs can be trapped ${ }^{19,20}$ and massive SWCNTs can be aggregated, ${ }^{21,22}$ but it is not easy to manipulate a single CNT; it is extremely difficult to optically trap a single nanoscale object, such as a single CNT, and simultaneously orient it. This difficulty arises because as the size of the trapped object decreases, the Brownian motion and the rotational diffusion coefficient will dramatically increase. ${ }^{23}$ Therefore, the controlled orientation and manipulation of a single CNT are challenging, and an optical method capable of achieving this objective is highly desirable. Recently, by using nanophotonic tweezers, a near-field optical method based on a precisely designed photonic crystal resonator that requires high accuracy in fabrication, single MWCNTs with outer diameters of 110-170 nm and a length of $5 \mu \mathrm{m}$ have been trapped and oriented. ${ }^{23}$ To arrange individual CNTs with controlled placement, after trapping and orientation, it is desirable to shift each trapped and oriented CNT to its target location while keeping its orientation unchanged. This requirement further increases the difficulties involved in the manipulation of a single CNT, particularly for CNTs of smaller sizes. Here, we show that, by using an optical-fiber nanotip, a single MWCNT of small size (e.g., an outer diameter of $50 \mathrm{~nm}$ and a length of $0.9 \mu \mathrm{m}$ ) undergoing large Brownian motion can be oriented and shifted under optical control while its orientation remains unchanged. The method requires only

State Key Laboratory of Optoelectronic Materials and Technologies, School of Physics and Engineering, Sun Yat-Sen University, Guangzhou 510275, China

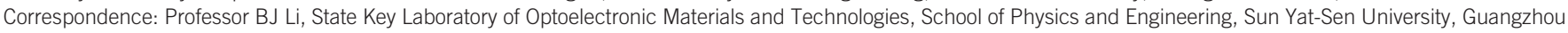
510275, China 
simple nanotip fabrication and can be used for the controllable orientation and shift of single MWCNTs of various sizes.

\section{MATERIALS AND METHODS}

\section{Fabrication of the optical-fiber nanotip}

The nanotip was fabricated by applying a flame-heating technique to a commercial single-mode optical fiber (connector type: FC/PC, core diameter: $9 \mathrm{~mm}$, cladding diameter: $125 \mathrm{~mm}$; Corning Inc.). The buffer and polymer jacket of the fiber were stripped off using a fiber stripper, and the fiber was sheathed with a glass capillary (inner diameter: $\sim 0.9 \mathrm{~mm}$, wall thickness: $\sim 0.1 \mathrm{~mm}$, length: $\sim 120 \mathrm{~mm}$ ) to protect the fiber from breakage and warping. The fiber was first heated for approximately $1 \mathrm{~min}$ until its melting point was reached. Then, an initial drawing speed of $0.5 \mathrm{~mm} \mathrm{~s}^{-1}$ was applied to the heated fiber, and the diameter of the fiber was decreased from $125 \mu \mathrm{m}$ to approximately $5 \mu \mathrm{m}$ over a 2-mm length to form a gradual taper. This was followed by the application of a drawing speed of approximately $5 \mathrm{~mm} \mathrm{~s}^{-1}$ to form an abruptly tapered region. The fiber was then broken to create a nanotip at its end by applying a drawing speed of approximately $2 \mathrm{~mm} \mathrm{~s}^{-1}$ after the formation of the abruptly tapered region. The nanotip at the end was formed by the surface tension of the melting fiber.

\section{Preparation of the MWCNT suspension}

An aqueous dispersion of MWCNTs (2.0 wt-\%) was purchased from a CNT supplier (XFNANO Inc., Nanjing, China). The purchased
MWCNTs were suspended in non-ionic surfactant to ensure that the MWCNTs were dispersed individually. The dispersion was diluted with deionized water to the desired concentration (the volume ratio of the dispersion to the added water was $\sim 1: 800$ ). To ensure that the MWCNTs were thoroughly dispersed, before the experiments, the MWCNT suspensions were shaken using an ultrasonic shaker for approximately $3 \mathrm{~min}$.

\section{RESULTS AND DISCUSSION}

Figure la presents a schematic illustration of the experimental setup. An optical fiber (sheathed with a glass capillary) is fixed and manipulated by a six-axis manipulator I (SAM I), the fiber nanotip is immersed in the aqueous suspension of MWCNTs, and a laser beam at a wavelength of $980 \mathrm{~nm}$ is launched into the nanotip for MWCNT manipulation. Because of their small size, individual MWCNTs are not visible under an optical microscope. To directly observe the manipulation of a single MWCNT, a blue laser beam at a wavelength of $473 \mathrm{~nm}$ is launched into another tapered optical fiber, the end of which is also immersed in the suspension (Supplementary Fig. S1). A microscope interfaced with a charged coupled device (CCD) camera through a personal computer is used to acquire the experimental data, including the manipulation dynamics of the MWCNTs. Figure $1 \mathrm{~b}$ schematically depicts the orientation and shifting of a single MWCNT using the nanotip and the 980-nm-wavelength laser beam. The MWCNT is first trapped by the end of the nanotip and is then
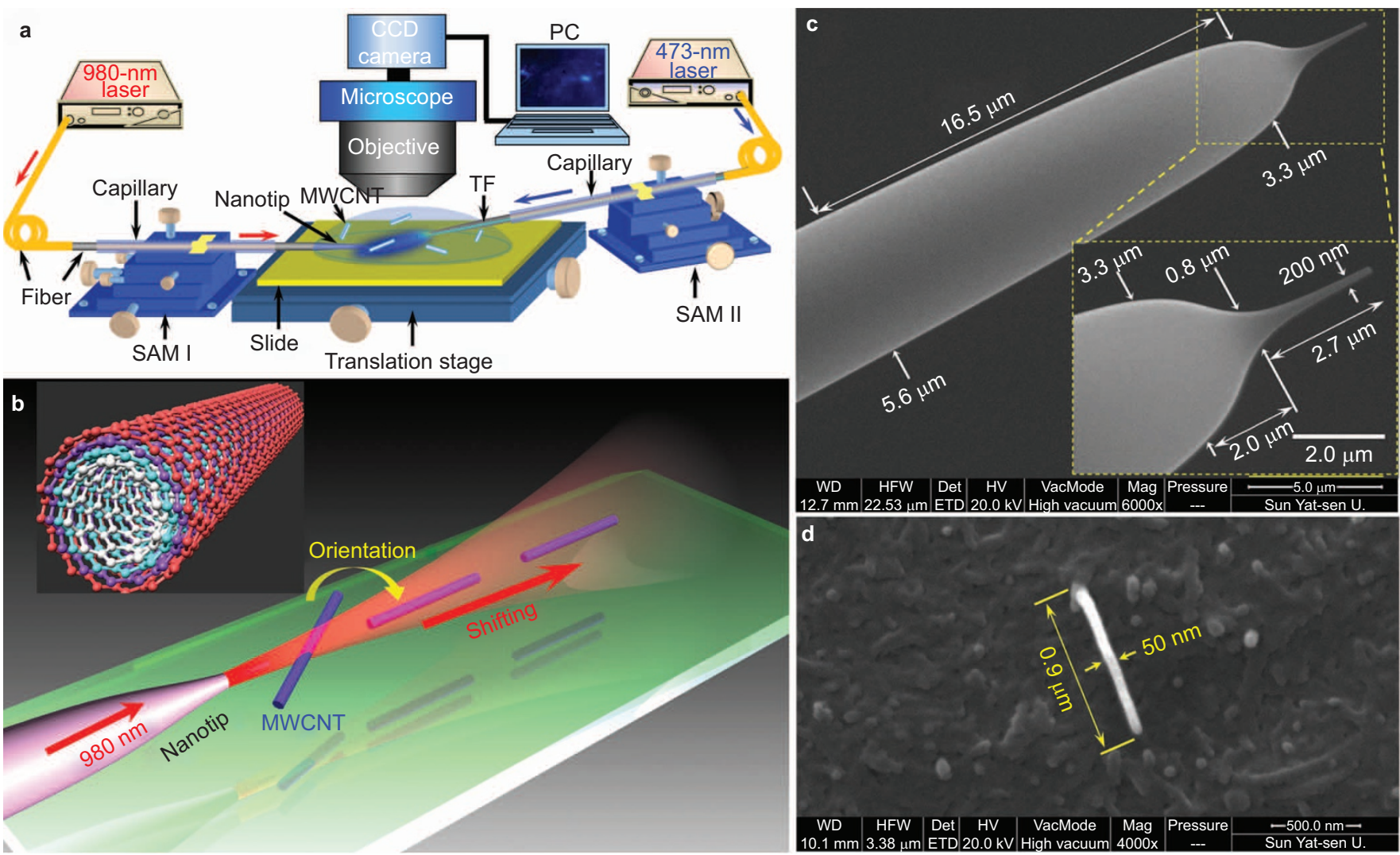

Figure 1 Experimental schematic and characterization of the nanotip and MWCNTs. (a) Schematic illustration of the experimental setup. The red and blue arrows indicate the propagation directions of the laser beams with wavelengths of 980 and $473 \mathrm{~nm}$, respectively. A suspension of MWCNTs is placed on a glass slide that is mounted and manipulated on a translation stage. (b) Schematic of the optical orientation and shifting of a single MWCNT using a laser beam at a wavelength of $980 \mathrm{~nm}$ launched into the nanotip. The inset schematically represents the structure of the MWCNT. (c) SEM image of the nanotip. The inset shows the details of the tapered region of the nanotip. (d) SEM image of a typical MWCNT. CCD, charge-coupled device; PC, personal computer; TF, tapered optical fiber; MWCNT, multiwalled carbon nanotube; SAM, six-axis manipulator; SEM, scanning electron microscope. 
oriented and shifted along the nanotip axis. Figure 1c presents an SEM image of the nanotip used in the experiments. The nanotip arm was characterized by a region in which the diameter decreased from 5.6 to $3.3 \mu \mathrm{m}$ over a length of $16.5 \mu \mathrm{m}$, followed by an abruptly tapered region in which the diameter decreased from 3.3 to $0.8 \mu \mathrm{m}$ over a length of $2.0 \mu \mathrm{m}$, which finally ended in a nanotip with a diameter that decreased from 800 to $200 \mathrm{~nm}$ over a length of $2.7 \mu \mathrm{m}$. Figure $1 \mathrm{~d}$ presents an SEM image of a typical single MWCNT with a length of $0.9 \mu \mathrm{m}$ and an outer diameter of $50 \mathrm{~nm}$.

The experiments were begun by launching the 473-nm-wavelength blue laser beam, with an optical power of $35 \mu \mathrm{W}$, into the tapered optical fiber. The optical force exerted on each MWCNT by this low optical power was too weak to affect the large Brownian motion of the MWCNT. Thus, the blue laser beam exerted no influence on the positions of the MWCNTs. After irradiation with the blue laser beam, the MWCNTs were then observed by means of the scattering of the blue light from the MWCNTs because of their large Rayleigh-scattering cross-section. ${ }^{24}$ The MWCNTs were found to be moving randomly in the suspension because of Brownian motion. Then, the 980-nm-wavelength laser beam was launched into the nanotip at various optical powers $(P)$ (measured at the output of the laser). The MWCNT motion was dominated by the competition among the optical forces, the Brownian motion, and viscous drag. The experimental results indicated that for $P<40 \mathrm{~mW}$, no MWCNTs could be trapped. For $P \geqslant 40 \mathrm{~mW}$, a MWCNT undergoing Brownian motion could be trapped by the tip because of the optical gradient force exerted by the tip; the MWCNT could then be oriented along the fiber axis by the exerted optical torque. During the process of orientation, the MWCNT was driven and shifted along the nanotip axis by the exerted optical scattering force. Figure $2 \mathrm{a}$ presents a bright-field optical microscope image of the nanotip immersed in the MWCNT suspension, showing the location of the nanotip. Figure $2 \mathrm{~b}$ schematically depicts the orientation angle $(\theta)$ and shift distance $(D)$ of the MWCNT along the nanotip axis. To illustrate the manipulation dynamics, as an example, Figure $2 c$ shows the orientation and shifting of a single MWCNT along the nanotip axis at $P=53 \mathrm{~mW}$. At $t=0$
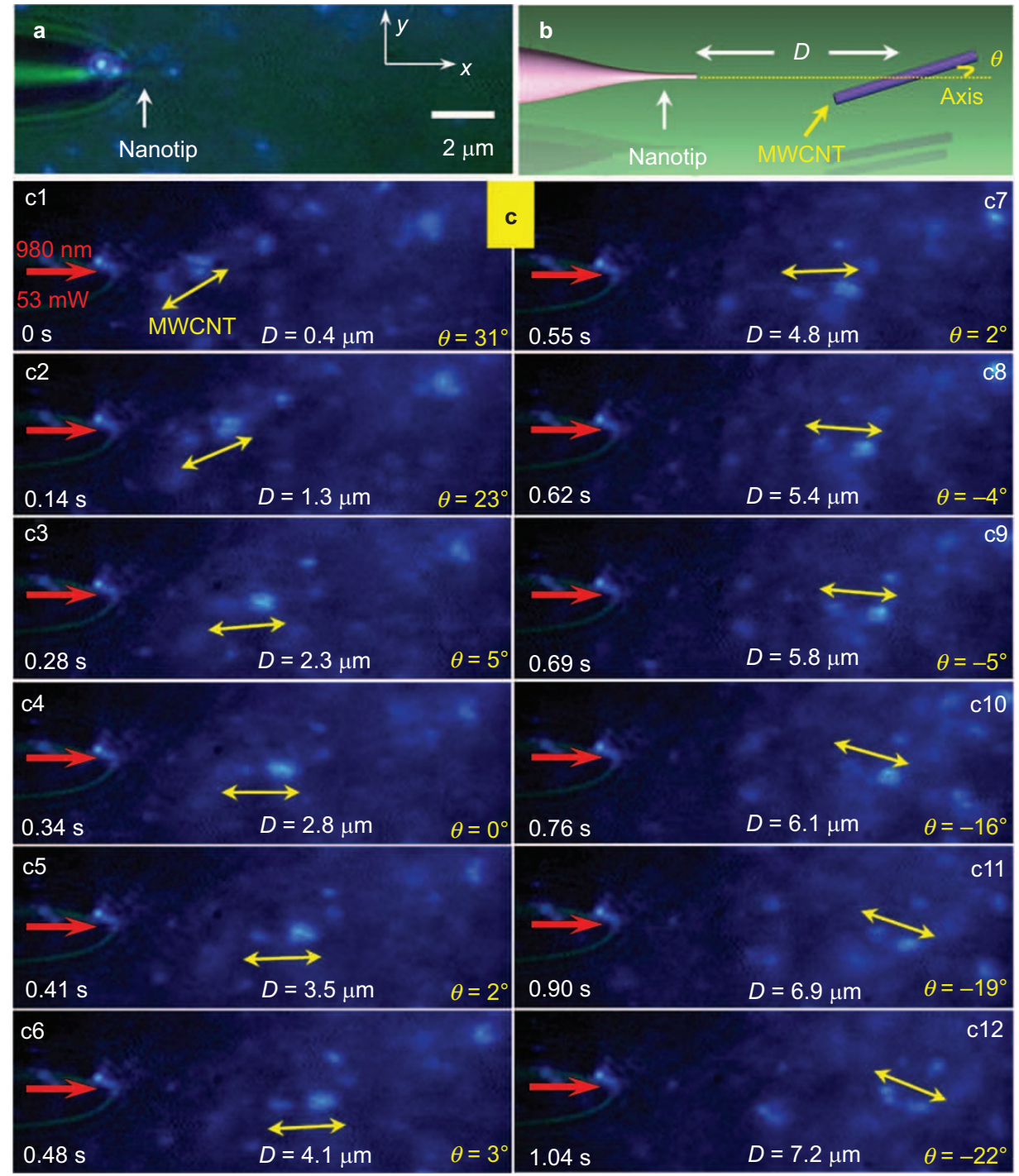

Figure 2 Optical orientation and shifting of a single MWCNT. (a) Bright-field optical microscope image of the nanotip. (b) Schematic diagram of the orientation and shifting of the MWCNT. The yellow dashed line represents the nanotip axis. (c) Dark-field optical microscope images showing the orientation and shift of a single MWCNT along the nanotip axis for a laser beam with an optical power of $53 \mathrm{~mW}$ launched into the nanotip. The red and yellow arrows indicate the input $980-\mathrm{nm}$ laser beam and the orientation of the MWCNT, respectively. MWCNT, multiwalled carbon nanotube. 
(Figure 2c1), the MWCNT is trapped near the nanotip, with $\theta=31^{\circ}$ and $D=0.4 \mu \mathrm{m}$. At $t=0.14 \mathrm{~s}$ (Figure 2c2), the MWCNT is oriented to $\theta=23^{\circ}$ and shifted to $D=1.3 \mu \mathrm{m}$. From $t=0.28$ to $0.69 \mathrm{~s}$ (Figure $2 \mathrm{c} 3-$ $2 \mathrm{c} 9), \theta$ is between $-5^{\circ}$ and $5^{\circ}$. In this range, the MWCNT can be regarded as being oriented along the nanotip axis with a deviation of $\pm 5^{\circ}$, which is caused by the Brownian motion during the shift. At $t=0.69 \mathrm{~s}$ (Figure 2c9), the MWCNT is shifted to $D=5.8 \mu \mathrm{m}$. After $t=0.69 \mathrm{~s}$, the orientation of the MWCNT gradually deviates from the nanotip axis with orientation angles of $\theta=-16^{\circ},-19^{\circ}$ and $-22^{\circ}$ and corresponding shift distances of $D=6.1,6.9$, and $7.2 \mu \mathrm{m}$, as shown in Figure $2 \mathrm{c} 10,2 \mathrm{c} 11$ and $2 \mathrm{c} 12$. This deviation is ascribed to the decrease in optical intensity along the nanotip axis, which causes the optical force to be gradually dominated by the Brownian motion. At $t=1.04 \mathrm{~s}$ (Figure 2c12), the motion of the MWCNT is totally dominated by the Brownian motion, and thus, the MWCNT is completely shifted by a distance of $7.2 \mu \mathrm{m}$ along the nanotip axis.

To illustrate the shifting of the MWCNT, Figure 3a presents the two-dimensional trajectory of the centroid of the MWCNT. Three different trajectory regions can be identified, as indicated by the labels I, II and III. In region I, the MWCNT is outside of the optical-trapping region, and thus, the MWCNT undergoes random Brownian motion. Once the MWCNT enters the optical-trapping region (region II), the trajectory changes to a straight route driven by the optical shift manipulation. In region III, because of the decrease in the optical intensity along the nanotip axis, the MWCNT motion has again become totally dominated by the Brownian motion, and the MWCNT therefore moves randomly. For direct comparison, as an example, Figure $3 \mathrm{~b}$ presents the trajectory of a free MWCNT that is not subjected to an optical shift. To verify that the free MWCNT is exhibiting Brownian motion, the mean squared displacement of the free MWCNT is plotted in Figure 3c; this plot demonstrates that the behavior of the mean squared displacement over time is highly linear, indicating that the free MWCNT is exhibiting Brownian motion. From Figure 3, it is
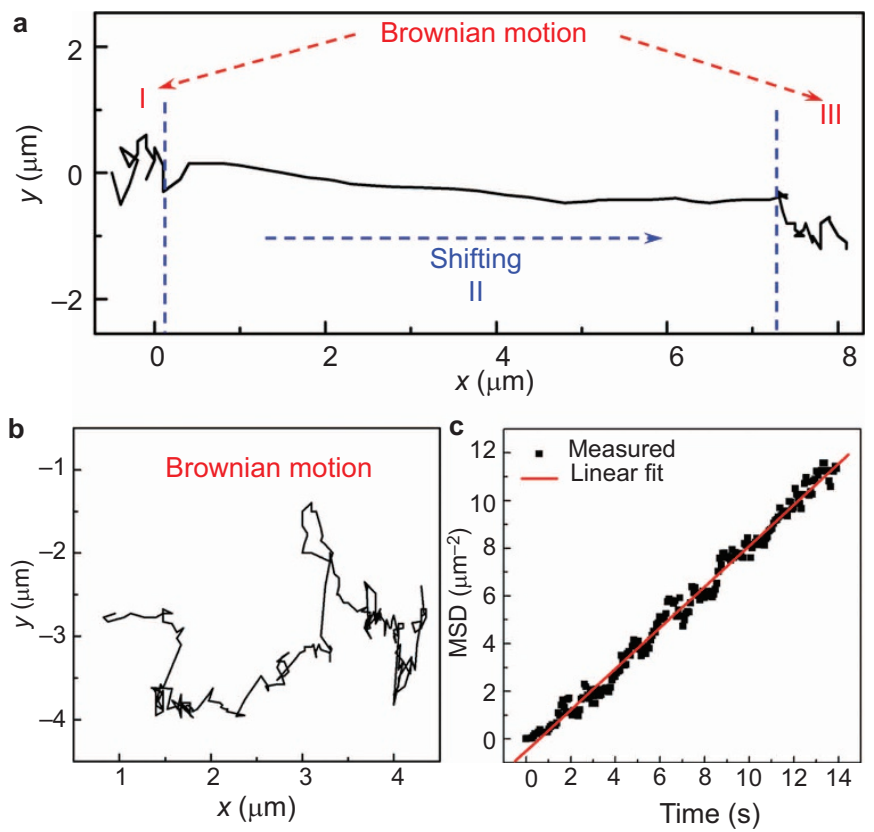

Figure 3 Trajectory of a single MWCNT. (a) In regions I and III, the MWCNT is undergoing Brownian motion, whereas in region II, the MWCNT is being shifted by the nanotip. (b) Trajectory of a free MWCNT. (c) MSD of the free MWCNT. MSD, mean squared displacement; MWCNT, multiwalled carbon nanotube. evident that the MWCNT is significantly influenced by the optical shift, thus allowing it to be shifted to a target location.

To further analyze the optical orientation and shifting of MWCNTs, a series of experiments were performed for different single MWCNTs in which laser beams at different optical powers $(P)$ were launched into the nanotip. Figure 4a presents the orientation angles $(\theta)$ of six single MWCNTs during the orientation process at $P=53 \mathrm{~mW}$. The laser was switched on at $t=0$. Once the optical power was applied, MWCNTs I-VI, which were undergoing Brownian motion with different orientation directions, were quickly oriented to approximately $\theta= \pm 5^{\circ}$ (region I in Figure 4a) within $0.27 \pm 0.02 \mathrm{~s}$. Then, the MWCNTs remained oriented at $\theta=0$ with a deviation of $\pm 5^{\circ}$ for a duration of $0.45 \pm 0.03 \mathrm{~s}$ (region II). Afterward, the orientations of the MWCNTs gradually deviated from the nanotip axis because the Brownian motion again began to dominate, and the MWCNTs became oriented in random directions (region III). For $P=53 \mathrm{~mW}$, the distance $\left(D_{\text {orie }}\right)$ required for the MWCNTs to become and remain oriented was $5.8 \pm 0.16 \mu \mathrm{m}$. $D_{\text {orie }}$ was observed to increase with increasing optical power (Figure $4 \mathrm{~b}$ ). Figure $4 \mathrm{c}$ shows the shift distance $(D)$ of each single MWCNT as a function of time. The largest $D$ values were $6.8,7.4$ and $8.0 \mu \mathrm{m}$ for $P=48,53$ and $60 \mathrm{~mW}$, respectively, with corresponding shift times of $1.17,1.10$ and $1.03 \mathrm{~s}$, respectively. After the shift, $D$ fluctuated because of Brownian motion. The experimental results indicated that the total shift distance $D_{\text {Total }}$ increased with increasing $P$, whereas the total shift time $(T)$ decreased with increasing $P$ (Figure $4 \mathrm{~d}$ ). This behavior occurred because the optical intensity (and thus the optical force) increased with increasing $P$. Therefore, the MWCNT was shifted more rapidly and over a greater distance for a larger $P$. The average shift velocity $(v)$ during the shift process was obtained using the equation $v=D_{\text {Total }} / T$ and is presented in Figure 4e. The fitted results indicate that $v$ increased nearly linearly with increasing $P$.

During the shift, the MWCNT motion is dominated by the competition among the optical force, the Brownian motion, and viscous drag. Because of the large aspect ratios and the variable shapes of the end facets of the MWCNTs, an MWCNT can be modeled as an elongated ellipsoid with a lateral hydrodynamic drag force $F_{\text {drag given by }}{ }^{17}$

$$
\left|\overrightarrow{\mathbf{F}}_{\mathrm{drag}}\right| \approx \frac{8 \pi \eta L}{2 \ln (2 L / d)+1} \cdot|\overrightarrow{\mathbf{v}}|
$$

where $\eta=8.9 \times 10^{-4} \mathrm{~Pa} \cdot \mathrm{s}$ is the viscosity of water at room temperature, $v$ is the average MWCNT shift velocity, and $L=0.9 \mu \mathrm{m}$ and $d=50 \mathrm{~nm}$ are the length and diameter of the MWCNT, respectively. Because the Brownian motion of the MWCNT is random and in random directions, the average applied optical force $\left(F_{\mathrm{O}}\right)$ can then be approximated to $F_{\text {drag. }}$. The calculated $F_{\mathrm{O}}$ is shown in Figure $4 \mathrm{e}$. The fitted result indicates that $F_{\mathrm{O}}$ increased nearly linearly with increasing $P$ because the optical force linearly increased with the increase in the optical intensity.

To demonstrate the orientation and shifting capability of the fiber nanotip for single MWCNTs of various sizes, experiments were conducted using four types of MWCNTs (samples A, B, C and D). Table 1 presents the sizes of the MWCNT samples. The volume $(V)$ of each MWCNT sample was estimated as $V=\pi d^{2} L / 4$, where $d$ and $L$ are the diameter and length of the MWCNT, respectively. Figure 5aI-5dI presents typical SEM images of samples A-D, respectively, whereas Figure 5aII-5dII presents typical dark-field optical microscope images of the orientation and shifting of the samples for an optical power of $53 \mathrm{~mW}$ launched into the fiber nanotip. With shift times of $t=0.52$, $0.48,0.58$ and $0.48 \mathrm{~s}$, the MWCNT samples A-D were shifted through 

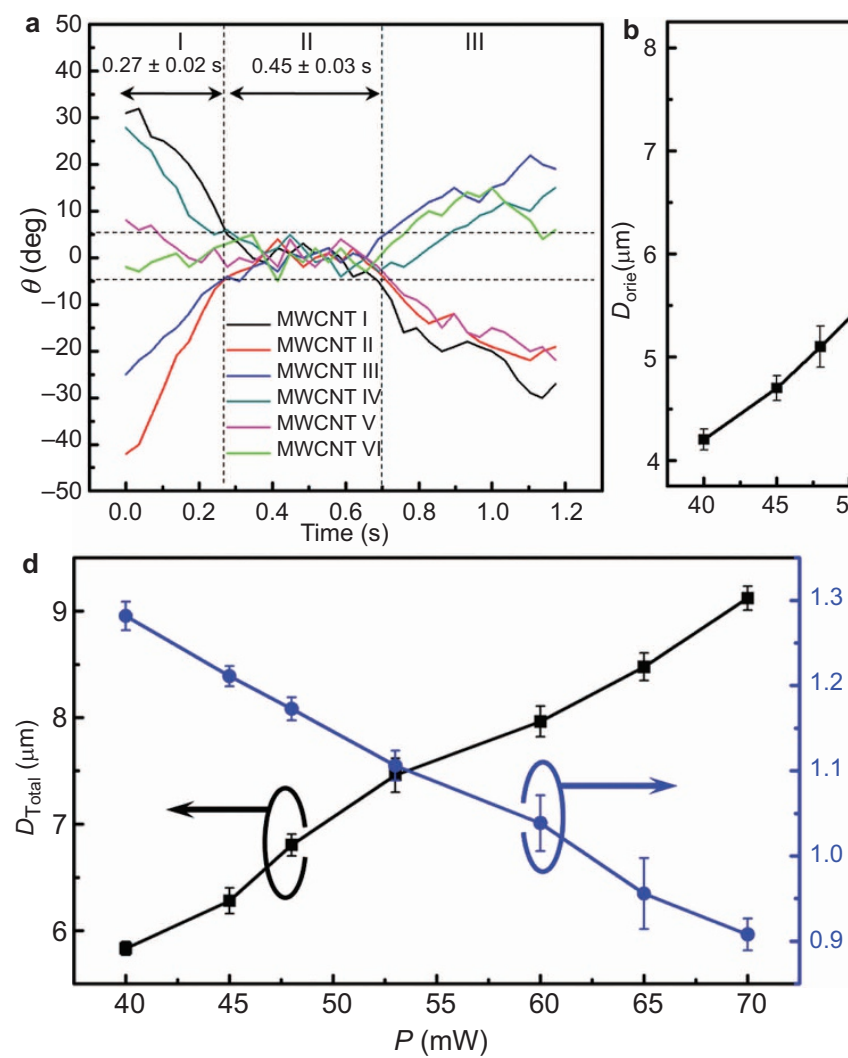
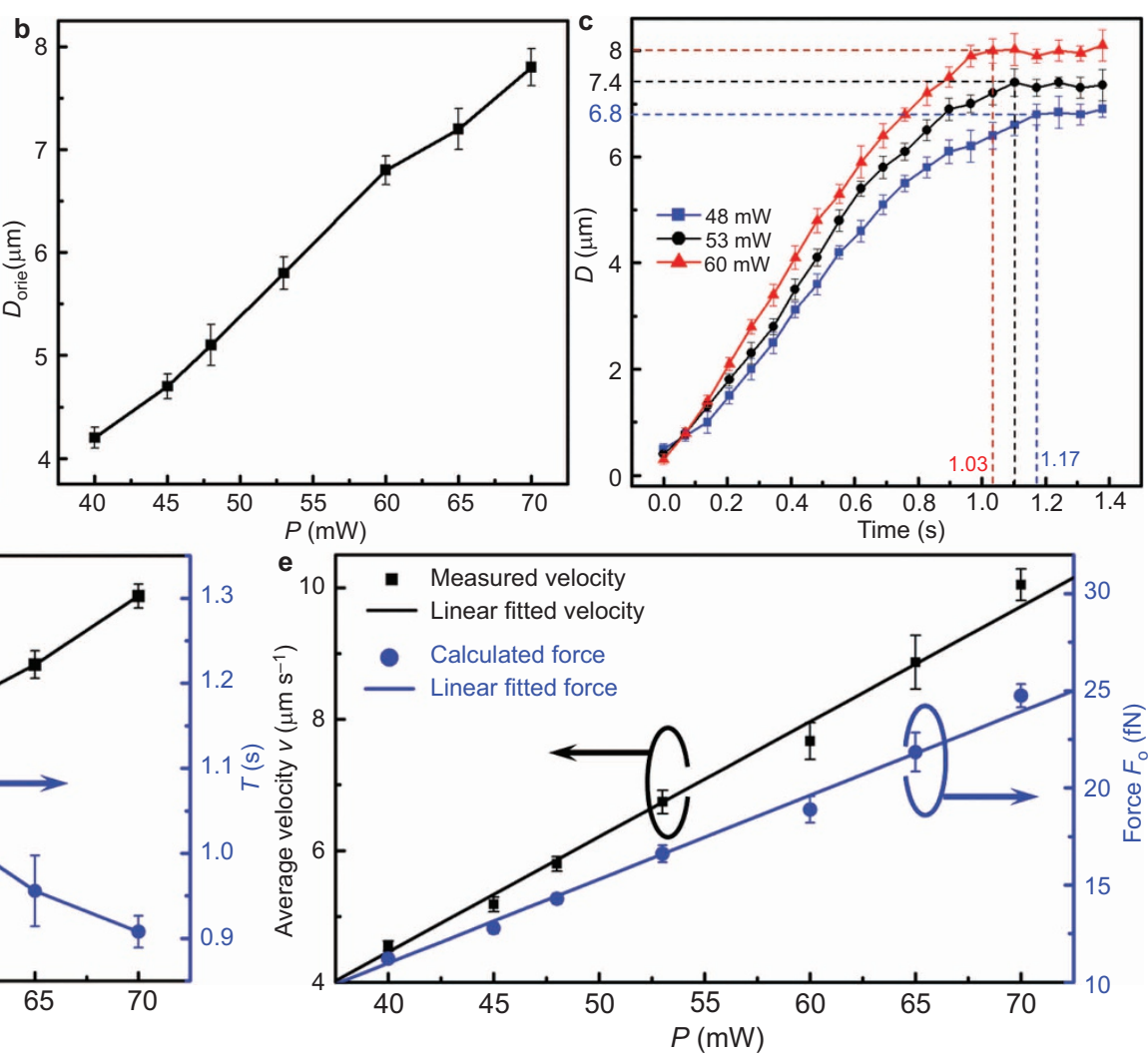

Figure 4 Optical orientation and shifting of single MWCNTs using different optical powers ( $P$ ). (a) Orientation angles $(\theta)$ as functions of time for different MWCNTs. (b) The distance $\left(D_{\text {orie }}\right)$ required for a single MWCNT to become and remain oriented by the laser as a function of the optical power $P$. (c) Shift distances $(D)$ for various optical powers as functions of time. (d) Total shift distance $\left(D_{\text {Total }}\right)$ and shift time $(T)$ as functions of the applied optical power. (e) Measured average shift velocity $(v)$ and calculated average optical force as functions of the applied power. MWCNT, multiwalled carbon nanotube.

distances of $D=5.2,4.1,5.0$ and $4.8 \mu \mathrm{m}$, respectively, along the nanotip axis. By analyzing the entire process of orientation and shifting, the average shift velocity $(v)$ was calculated as a function of the MWCNT volume (Figure 5e). Using Equation (1), the average optical force $\left(F_{\mathrm{O}}\right)$ over the entire shift process was also calculated (Figure $5 \mathrm{f}$ ). It is evident that $F_{\mathrm{O}}$ increased with increasing MWCNT volume. This increase was not linear and will be discussed below in relation to the optical-force analysis.

To more precisely control the orientation and shifting of a single MWCNT, a dual-fiber-nanotip method (see Supplementary Fig. S2 for images of the nanotips) was used, as schematically illustrated in Figure 6a. When a single MWCNT is trapped by nanotip I at an optical power of $P_{1}$ (980-nm wavelength) and nanotip II at an optical power of $P_{2}$ (980-nm wavelength), the location of the MWCNT can be controlled by adjusting the distance between the two nanotips and/or the optical powers of the laser beams launched into the nanotips. For example, Figure $6 \mathrm{~b}$ and $6 \mathrm{c}$ presents dark-field images of the orientation and shifting of single MWCNTs with $P_{2}=53$ and $61 \mathrm{~mW}$, respectively, launched into nanotip II with $P_{1}$ remaining equal to $53 \mathrm{~mW}$. The distance between the two nanotips was $7.0 \mu \mathrm{m}$. Figure $6 \mathrm{~b}$ illustrates

Table 1 Sizes of various multiwalled carbon nanotube samples used to investigate optical orientation and shifting capabilities

\begin{tabular}{lcccc}
\hline Sample & Length $L(\mu \mathrm{m})$ & Diameter $d(\mathrm{~nm})$ & Aspect ratio & Volume $V\left(\times 10^{6} \mathrm{~nm}^{3}\right)$ \\
\hline A & $0.68 \pm 0.018$ & $70 \pm 7.2$ & 9.7 & 2.6156 \\
B & $0.72 \pm 0.022$ & $60 \pm 4.8$ & 12.0 & 2.0347 \\
C & $0.90 \pm 0.035$ & $50 \pm 5.3$ & 18.0 & 1.7663 \\
D & $1.0 \pm 0.030$ & $65 \pm 4.6$ & 15.4 & 3.3166 \\
\hline
\end{tabular}

that for $P_{1}=P_{2}=53 \mathrm{~mW}$, the MWCNT was oriented and located at a distance $\left(D_{1}\right)$ of $3.4 \mu \mathrm{m}$ from nanotip I. When $P_{2}$ was increased to $61 \mathrm{~mW}, D_{1}$ decreased to $2.3 \mu \mathrm{m}$ (Figure $6 \mathrm{c}$ ). Figure $6 \mathrm{~d}$ shows the distance $\left(D_{1}\right)$ between the MWCNT and nanotip I for various $P_{2}$ values; the relation is highly linear. Therefore, the precise orientation and shift control of a single MWCNT can be achieved by adjusting the distance between the two nanotips and the optical powers of the laser beams applied to them.

The optical trapping of the MWCNT by the nanotip and the orientation process can be ascribed to the interaction between the electric-field components (E) of the trapping laser and the instantaneous dipole moments $(\mathbf{P})$. The dipolar polarizability determines the strength of the interaction with the optical field. ${ }^{25}$ The induced dipole moment per unit volume is $\mathbf{P}=\varepsilon_{0} \chi \mathbf{E}$, where $\varepsilon_{0}$ and $\chi$ are the permittivity of free space and the dielectric susceptibility, respectively. The interaction energy for the MWCNT is then given by ${ }^{21}$

$$
U=-\langle\mathbf{P} \cdot \mathbf{E}\rangle / 2=-\varepsilon_{0} \chi\langle\mathbf{E}\rangle^{2} / 2
$$

The interaction results in a force that is determined by the gradient of the laser intensity. A force in the same direction as the opticalintensity gradient can trap an MWCNT in the region where the laser intensity is higher, and thus, an MWCNT near the nanotip can be trapped by the transverse optical gradient force perpendicular to the fiber axis. However, for an MWCNT at the fiber axis, this gradient force is smaller than the scattering force induced by the photon pressure, and thus, the MWCNT is shifted along the fiber axis by the optical scattering force, which is given $b^{26}$ 


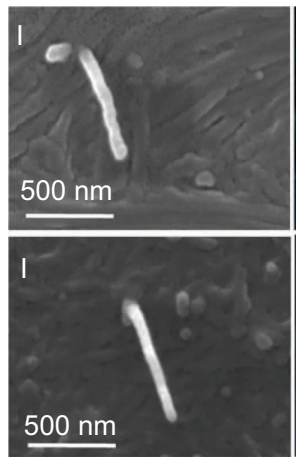

C

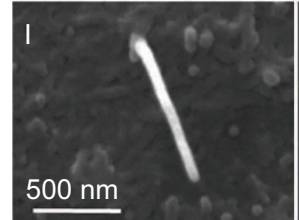

d
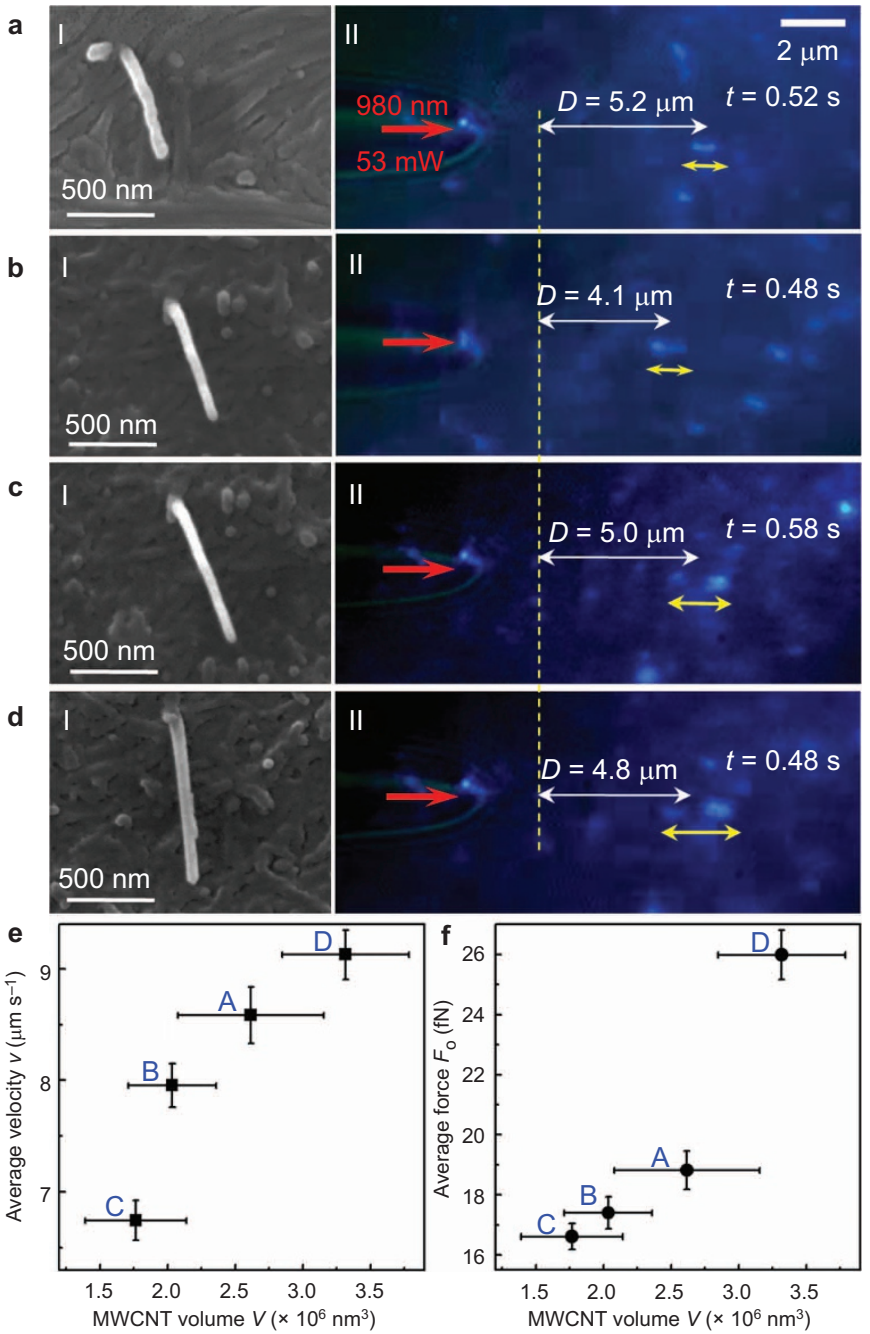

Figure 5 Optical orientation and shifting of various single MWCNTs. (a-d) Panels I: SEM images of MWCNT samples A-D. Panels II: dark-field optical microscope images of the orientation and shifting of MWCNT samples A-D. The yellow dashed line, red arrows, and yellow arrows indicate the end of the nanotip, the launched $980-\mathrm{nm}$ laser beams (53 mW), and the orientations of the MWCNTs, respectively. (e) Measured average shift velocity $(v)$ as a function of the CNT volume $(V$ ). The letters A-D represent the four MWCNT samples. (f) Calculated average optical force $\left(F_{O}\right)$ as a function of the MWCNT volume $(V)$. MWCNT, multiwalled carbon nanotube; SEM, scanning electron microscope.

$$
\mathbf{F}_{\text {scat }}=\frac{n_{\mathrm{w}}}{c}\langle\mathbf{S}\rangle C_{\text {scat }}
$$

where $n_{\mathrm{w}}$ is the refractive index of water, $c$ is the speed of light, $\mathbf{S}$ is the Poynting vector and $C_{\text {scat }}=k^{4}|\alpha|^{2} /(4 \pi)$ is the scattering cross-section; here, $\alpha$ is the polarizability of the MWCNT, which is given by $\alpha=V\left(\varepsilon_{\mathrm{C}^{-}}\right.$ $\left.\varepsilon_{\mathrm{w}}\right) /\left(\varepsilon_{\mathrm{C}}+2 \varepsilon_{\mathrm{W}}\right)$, where $V$ is the MWCNT volume and $\varepsilon_{\mathrm{C}}$ and $\varepsilon_{\mathrm{w}}$ are the dielectric constants of the MWCNT and water, respectively. Because of the unique optical and electronic properties of MWCNTs, the dielectric constant of an MWCNT depends on its size; ${ }^{27}$ thus, there is no simple linear relation between $\alpha$ and the MWCNT volume $V$. From Equation (3) and the expression for $\alpha$, it is apparent that $F_{\text {scat }}$ increases as the MWCNT volume $V$ increases, but not in a linear manner. Therefore, the experimental estimation of the average optical forces exerted on MWCNTs with different volumes using Equation (1) is not characterized by a simple, linearly increasing relation, as shown in Figure 5f.
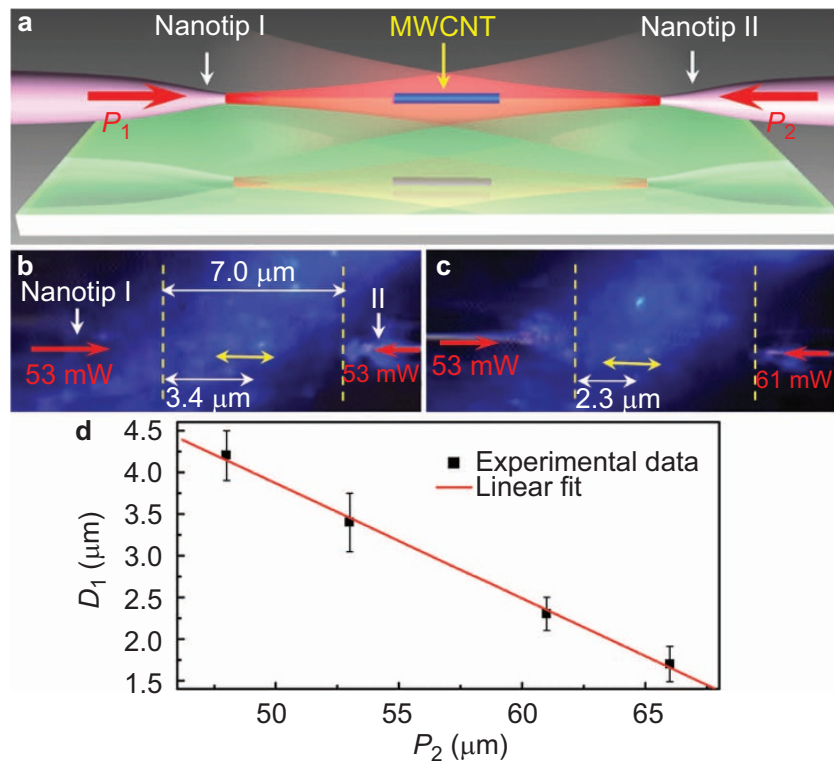

Figure 6 Controlling the orientation and shift of a single MWCNT using a dualfiber-nanotip method. (a) Schematic depiction of the dual-fiber-nanotip method; the two red arrows indicate the laser beams launched into nanotips I and II. (b, c Dark-field optical microscope images of the orientation and shifting of a single MWCNT using (b) $P_{1}=P_{2}=53 \mathrm{~mW}$ and (c) $P_{1}=53 \mathrm{~mW}$ and $P_{2}=61 \mathrm{~mW}$. The yellow dashed lines indicate the locations of the two nanotip ends, whereas the yellow arrows indicate the orientations of the single MWCNTs. (d) Measured $D_{1}$ as a function of $P_{2}$. MWCNT, multiwalled carbon nanotube.

For an electromagnetic wave with a transverse electric mode, when an MWCNT with an orientation angle of $\theta$ with respect to the nanotip axis is trapped on the nanotip axis, a torque is generated, whose magnitude is given by $^{23}$

$$
\mathbf{T}_{0}=1 / 2 \operatorname{Re}\left[\mathbf{P} \times \mathbf{E}^{*}\right]
$$

where * represents a complex conjugate and $\operatorname{Re}[]$ indicates the real part of the expression inside the brackets. For an MWCNT with an orientation of $\theta$ with respect to the nanotip axis, considering the effective polarizability of the MWCNT, the induced torque can be expressed as follows: ${ }^{23}$

$$
T=1 / 2 \alpha_{\mathrm{eff}}|\mathbf{E}|^{2} \sin \theta \cos \theta
$$

where $\alpha_{\text {eff }}=\operatorname{Re}\left[\boldsymbol{\alpha}_{\text {long }}-\boldsymbol{\alpha}_{\text {trans }}\right]$ is the effective polarizability; here, $\boldsymbol{\alpha}_{\text {long }}$ and $\boldsymbol{\alpha}_{\text {trans }}$ are the polarizabilities of the ellipsoid along the longitudinal and transverse axes, respectively. Thus, the MWCNT is finally oriented along the nanotip axis such that $\theta=0$. For this orientation, the overall polarizability, which depends on the overlap of the light intensity and the MWCNT volume, is maximized in an optical trap. ${ }^{28}$ As a result, the MWCNT is oriented along the optical axis of the nanotip. However, because of the influence of Brownian motion, deviations will arise, as observed in the experiments. The $|\mathbf{E}|^{2}$ value output by the nanotip can be obtained using a finite-element method. The simulation model is depicted in Supplementary Fig. S3, and the simulated $|\mathbf{E}|^{2}$ values are presented in Supplementary Fig. S4. Simulation results also indicate that the maximum value of $|\mathbf{E}|^{2}$ output by the nanotip along the fiber axis is 1.5 times the value output by a tapered optical fiber without a nanotip. Thus, a fiber with a nanotip is more suitable for 
the orientation and shifting of MWCNTs. However, because of the possible surface plasmon resonances and different chiralities of MWCNTs in different chemical environments, the dielectric constants and conductivities of MWCNTs are difficult to obtain. ${ }^{23}$ Thus, the optical model for the MWCNT simulation, from which numerical calculations of the optical forces and optical torque can be obtained, is difficult to construct.

Recent studies have demonstrated the remarkable ability to orient single nanoscale rod-shaped objects, such as gold nanorods, using classical optical tweezers (COTs) ${ }^{26}$ and the ability to simultaneously orient multiple rod-shaped bacteria using holographic optical tweezers (HOTs); ${ }^{29}$ however, compared to the COTs and HOTs methods, methods of trapping and manipulation using one or more opticalfiber nanotips offer the following advantages: (i) an optical-fiber nanotip can be used to trap and manipulate nanoscale particles with only a single optical fiber used to deliver laser beams, whereas the COTs and HOTs methods require the bulky structure of a highnumerical-aperture focusing objective and optical system to deliver the laser beams; ${ }^{30}$ and (ii) the low working distance of the highnumerical-aperture focusing objective in such a COTs or HOTs setup limits the depth at which particles can be manipulated and analyzed, whereas, when a flexible fiber nanotip is used, particles can be easily manipulated at various depths in the suspension because the fiber can be inserted into the particle suspension in various directions and to various depths. ${ }^{30,31}$ Therefore, the optical-fiber nanotip used in our work may provide the ability to orient and manipulate various types of nanoscale objects.

Additional experiments demonstrated that when polarized laser beams are launched into the optical-fiber nanotip, the orientation process for the MWCNTs is no different than in the case of orientation using non-polarized laser beams. This polarization insensitivity may be attributable to the fact that the bending of the fiber and the tapered end of the fiber fabricated via the flame-heating method strongly influence the polarization of the propagating laser beams. Thus, after the beam has propagated along the fiber, the initial polarization of the laser beam is not maintained in the beam that is output by the nanotip. In comparison with the orientation of nanoscale rod-shaped objects using COTs, in which different orientations of the objects can be achieved using differently polarized laser beams, this is a disadvantage of our method for the orientation of MWCNTs. Although we have used tapered optical fiber to manipulate various objects, ${ }^{30,32,33}$ the objects that can be thus trapped and manipulated are limited to microscale/submicroscale dielectric particles (the smallest are $0.7-\mu \mathrm{m} \mathrm{SiO}_{2}$ particles) and microscale/submicroscale biological cells (the smallest are Escherichia coli cells, with a diameter of approximately $700 \mathrm{~nm}$ and a length of approximately $2.0 \mu \mathrm{m}$ ). These objects can be easily observed using an optical microscope, and their Brownian motion is not significant because of their large sizes. Therefore, the advancement represented by this work is that a single MWCNT with a diameter as small as $50 \mathrm{~nm}$ can now be trapped, precisely oriented, and shifted to a designated position.

\section{CONCLUSIONS}

In conclusion, we demonstrated an optical method capable of the controlled orientation and targeted shifting of single MWCNTs using an optical-fiber nanotip. When an optical power of greater than $40 \mathrm{~mW}$ was launched into the nanotip, a single MWCNT with an outer diameter of only $50 \mathrm{~nm}$ and a length of $0.9 \mu \mathrm{m}$ was trapped by the nanotip and then oriented and shifted along the fiber axis by overcoming its considerable Brownian motion. The orientation angles and shift distances for various optical powers were characterized, and the average optical force exerted on the MWCNT in the shifting process was estimated. This orientation and shifting capability was also demonstrated for MWCNTs of various sizes. The ability to orient and shift a single MWCNT provides a new approach to the manipulation of one-dimensional materials with controlled arrangements and should facilitate the development of high-precision assemblies of MWCNTs.

\section{ACKNOWLEDGEMENTS}

The authors thank Hao Chen and Qiuguo Li from Sun Yat-Sen University for fruitful discussions and Yuchao Li from Sun Yat-Sen University for experimental assistance in the preparation of the revised manuscript. This work was supported by the National Natural Science Foundation of China (Nos. 11274395 and 61205165), the Program for Changjiang Scholars and Innovative Research Team in University (IRT13042) and the 2012 New Academic Researcher Award for Doctoral Candidates granted by the Ministry of Education, China.

1 Hu L, Hecht DS, Grüner G. Carbon nanotube thin films: fabrication, properties, and applications. Chem Rev 2010; 110: 5790-5844.

2 De Volder MF, Tawfick SH, Baughman RH, Hart AJ. Carbon nanotubes: present and future commercial applications. Science 2013; 339: 535-539.

3 Shekhar S, Stokes P, Khondaker SI. Ultrahigh density alignment of carbon nanotube arrays by dielectrophoresis. ACS Nano 2011; 5: 1739-1746.

4 Cao Q, Han SJ, Tulevski GS, Zhu Y, Lu DD et al. Arrays of single-walled carbon nanotubes with full surface coverage for high-performance electronics. Nat Nanotechnol 2013; 8: 180-186.

5 Liu Z, Jiao L, Yao Y, Xian X, Zhang J. Aligned, ultralong single-walled carbon nanotubes: from synthesis, sorting, to electronic devices. Adv Mater 2010; 22: 2285-2310.

6 Purcell ST, Vincent $P$, Journet $C$, Binh VT. Hot nanotubes: stable heating of individua multiwall carbon nanotubes to $2000 \mathrm{~K}$ induced by the field-emission current. Phys Rev Lett 2002; 88: 105502.

7 Hinds BJ, Chopra N, Rantell T, Andrews R, Gavalas V et al. Aligned multiwalled carbon nanotube membranes. Science 2004; 303: 62-65.

8 Liu H, Takagi D, Chiashi S, Homma Y. Transfer and alignment of random single-walled carbon nanotube films by contact printing. ACS Nano 2010; 4: 933-938.

9 Wang Y, Maspoch D, Zou S, Schatz GC, Smalley RE et al. Controlling the shape, orientation, and linkage of carbon nanotube features with nano affinity templates. Proc Natl Acad Sci USA 2006; 103: 2026-2031.

10 Jiao L, Xian X, Wu Z, Zhang J, Liu Z. Selective positioning and integration of individual single-walled carbon nanotubes. Nano Lett 2009; 9: 205-209.

11 Gao B, Duan X, Zhang J, Wu T, Son $\mathrm{H}$ et al. Raman spectral probing of electronic transition energy $E_{\mathrm{ii}}$ variation of individual SWCNTs under torsional strain. Nano Lett 2007; 7: 750-753.

12 Khang DY, Xiao J, Kocabas C, MacLaren S, Banks T et al. Molecular scale buckling mechanics in individual aligned single-wall carbon nanotubes on elastomeric substrates. Nano Lett 2008; 8: 124-130.

13 Peng B, Locascio M, Zapol P, Li S, Mielke SL et al. Measurements of near-ultimate strength for multiwalled carbon nanotubes and irradiation-induced crosslinking improvements. Nat Nanotechnol 2008: 3: 626-631.

14 Zhang R, Ning Z, Zhang Y, Xie H, Zhang Q et al. Facile manipulation of individua carbon nanotubes assisted by inorganic nanoparticles. Nanoscale 2013; 5: 65846588.

15 Krupke R, Hennrich F, Löhneysen HV, Kappes MM. Separation of metallic from semiconducting single-walled carbon nanotubes. Science 2003; 301: 344-347.

16 Vijayaraghavan A, Blatt S, Weissenberger D, Oron-Carl M, Hennrich F et al. Ultra-largescale directed assembly of single-walled carbon nanotube devices. Nano Lett 2007; 7: 1556-1560.

17 Pauzauskie PJ, Jamshidi A, Valley JK, Satcher JH, Wu MC. Parallel trapping of multiwalled carbon nanotubes with optoelectronic tweezers. Appl Phys Lett 2009; 95: 113104.

18 Maragò OM, Jones PH, Gucciardi PG, Volpe G, Ferrari AC. Optical trapping and manipulation of nanostructures. Nat Nanotechnol 2013; 8: 807-819.

19 Zhang J, Kim HI, Oh CH, Sun X, Lee H. Multidimensional manipulation of carbon nanotube bundles with optical tweezers. Appl Phys Lett 2006; 88: 053123.

20 Maragò OM, Jones PH, Bonaccorso F, Scardaci V, Gucciardi PG et al. Femtonewton force sensing with optically trapped nanotubes. Nano Lett 2008; 8: 3211-3216.

21 Tan S, Lopez HA, Cai CW, Zhang Y. Optical trapping of single-walled carbon nanotubes. Nano Lett 2004; 4: 1415-1419.

22 Rodgers T, Shoji S, Sekkat Z, Kawata S. Selective aggregation of single-walled carbon nanotubes using the large optical field gradient of a focused laser beam. Phys Rev Lett 2008; 101: 127402 . 
23 Kang P, Serey X, Chen YF, Erickson D. Angular orientation of nanorods using nanophotonic tweezers. Nano Lett 2012; 12: 6400-6407.

24 Yu Z, Brus L. Rayleigh and Raman scattering from individual carbon nanotube bundles. J Phys Chem B 2001; 105: 1123-1134.

25 Gordon JP. Radiation forces and momenta in dielectric media. Phys Rev A 1973; 8: 14-21.

26 Selhuber-Unkel C, Zins I, Schubert O, Sönnichsen C, Oddershede LB. Quantitative optical trapping of single gold nanorods. Nano Lett 2008; 8: 2998-3003.

27 Kataura H, Kumazawa Y, Maniwa Y, Umezu I, Suzuki S et al. Optical properties of single-wall carbon nanotubes. Synth Met 1999; 103: 2555-2558.

28 Koch M, Rohrbach A. Object-adapted optical trapping and shape-tracking of energyswitching helical bacteria. Nat Photonics 2012; 6: 680-686.

29 Hörner F, Woerdemann M, Müller S, Maier B, Denz C. Full 3D translational and rotational optical control of multiple rod-shaped bacteria. J Biophoton 2010; 3: $468-475$.

30 Xin H, Xu R, Li B. Optical trapping, driving, and arrangement of particles using a tapered fibre probe. Sci Rep 2012; 2: 818.
31 Mohanty S. Optically-actuated translational and rotational motion at the microscale for microfluidic manipulation and characterization. Lab Chip 2012; 12: 3624-3636.

32 Xin H, Li Y, Li L, Xu R, Li B. Optofluidic manipulation of Escherichia coli in microfluidic channel using an abruptly tapered optical fiber. Appl Phys Lett 2013; 103: 033703.

33 Xin H, Zhang Y, Lei H, Li Y, Zhang H et al. Optofluidic realization and retaining of cellcell contact using an abrupt tapered optical fibre. Sci Rep 2013; 3: 1993.

(c) (i) $\odot$ This work is licensed under a Creative Commons Attribution-

c. party material in this article are included in the article's Creative Commons license, unless indicated otherwise in the credit line; if the material is not included under the Creative

Commons license, users will need to obtain permission from the license holder to reproduce the material. To view a copy of this license, visit http://creativecommons.org/licenses/ by-nc-nd/3.0/

Supplementary information for this article can be found on the Light: Science \& Applications' website (http://www.nature.com/lsa/). 